\title{
Reactions of Cyclobutenones with Nucleophilic Reagents via Vinylketen Intermediates
}

\author{
By Rolf Huisgen* and Herbert Mayr
}

(Institut für Organische Chemie der Universität, 8 München 2, Karlstr. 23, Germany)

Summary While strong nucleophiles add to the endocyclic double bond of cyclobutenones to yield cyclobutanones, weaker nucleophiles (e.g. alcohols, aniline) interact with the vinylketens which are thermally or photochemically generated from cyclobutenones; the alcohol adducts reveal the steric course of this ring opening which is virtually irreversible for phenylated and alkylated cyclobutenones.

Chlorinated cyclobutenones equilibrate with small concentrations of vinylketens as shown by trapping reactions with nucleophiles. ${ }^{1}$ Recently, phenylated and alkylated cyclobutenones have become readily obtainable by aluminainduced alcohol elimination from 3-alkoxycyclobutanones

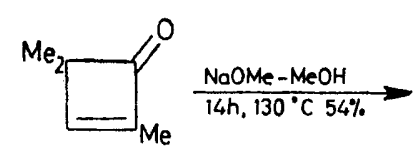

(1)

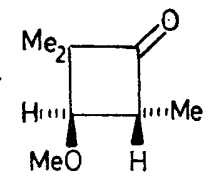

(2)

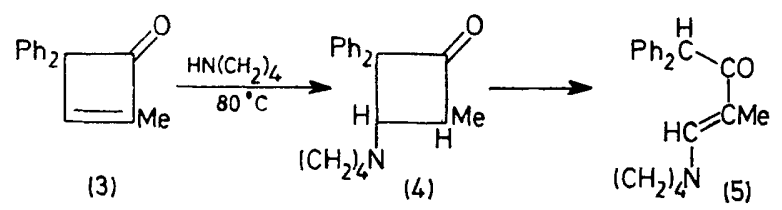

(adducts from ketens and enol ethers). ${ }^{2}$ The isolated products formed from the thermolysis of 2,4-dimethyl-4-phenylcyclobut-2-enone suggested a mechanistic pathway in which an irreversible ring opening to cis-trans isomeric vinylketens takes place. ${ }^{3}$

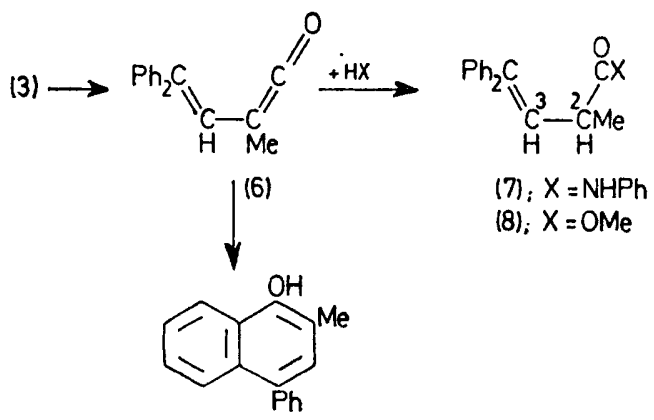

(9)

The electrophilic $\mathrm{C}=\mathrm{C}$ double bond of cyclobutenones is capable of adding nucleophilic reagents. The reaction of the trimethylcyclobutenone (1) with a $\mathrm{MeOH}-\mathrm{NaOMe}$ solution resulted in the formation of the trans-cyclobutanone (2) and a trace of the cis-isomer; the observed i.r. frequency at $1771 \mathrm{~cm}^{-1}$ and the ${ }^{1} \mathrm{H}$ n.m.r. parameters ${ }^{4}$ establish the structure (2).

The formation of $64 \%$ (5) from the reaction of (3) with pyrrolidine in refluxing benzene suggests the intermediacy of (4) which could then undergo the well-known base- 
catalysed ring opening of 3-aminocyclobutanones to 2acylenamines. ${ }^{5}$ The strong i.r. absorptions at 1560 and $1622 \mathrm{~cm}^{-1}$ as well as the vinyl-H signal at $\tau 2.40$ indicate the $\beta$-acylenamine structure (5).

However, aniline at $100^{\circ} \mathrm{C}$ did not attack (3), but rather intercepted the vinylketen (6) which emerges from the thermal electrocyclic ring opening of (3). The anilide (7), isolated in $75 \%$ yield, is indicated to be the but-3-enoic acid derivative on the basis of its n.m.r. spectrum, $\tau \mathbf{3 \cdot 8 0}$ $\left(d, J_{2,3} 10 \mathrm{~Hz}, 3-\mathrm{H}\right.$ ), $6 \cdot 80$ (doublet of quadruplets, $J_{2}$, Me $6.8 \mathrm{~Hz}, 2-\mathrm{H})$, and $8.68(\mathrm{~d}, 2-\mathrm{Me})]$. Also the formation of (8) from the reaction of (3) and methanol provides evidence for the intermediacy of (6). The spectra of (8) again establish the 1,2-addition of the nucleophilic reagent to the vinylketen (6).

In addition to the ester (8) the reaction yields the naphth1-ol (9) which is formed quantitatively from (3) in boiling benzene. $^{3}$ The ratio of these competing reactions in $\mathrm{MeOH}$, i.e., (8): (9), is 8.4 at $25{ }^{\circ} \mathrm{C}$ (reaction time 14 days) and 1.8 at $80^{\circ} \mathrm{C}(1 \mathrm{~h})$. The smaller temperature coefficient of the ester formation stems from its relatively large negative activation entropy ${ }^{6}$ and the ratio (8):(9) reflects the competition between an intermolecular and an intramolecular process. Similar products were obtained in the methanolysis of 4,4-diphenylcyclobut-2-enone and its 2-ethyl derivative.

The electrocyclic ring opening of 2,4-dimethyl-4-phenylcyclobut-2-enone (10) provides the cis-trans isomeric vinylketens (11) and (12); only (11) is capable of undergoing intramolecular electrophilic aromatic substitution to give the naphth-1-ol (13). Methanol is again observed to add to the 1,2 positions of (11) and (12). The n.m.r. spectra of the methyl esters (14) and (15) are consistent only with a $\beta, \gamma$ unsaturated structure; $\tau(3-\mathrm{H}) \quad 4 \cdot 47$ for the

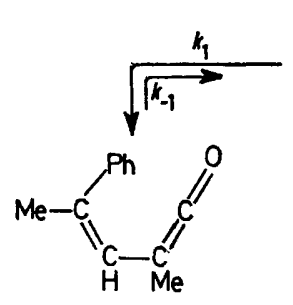

(11) $\downarrow_{3} k_{2} \downarrow+\mathrm{MeOH}$

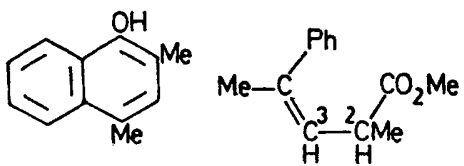

(13)

(10)
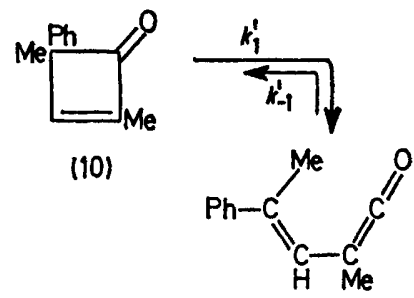

(12)
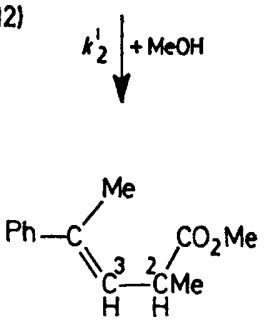

(15)
$(Z)$-ester (14) and 4.21 for the $(E)$-ester (15) compared with 4.64 and 4.20 calculated from substituent increments.

Reaction of (10) with methanol at $80^{\circ} \mathrm{C}$ provided $24 \%$ naphthol (13), 6\% ester (14) and 70\% ester (15). When the thermolysis of (10) was carried out in a solution of methanol in benzene $(0.6-4.6 \mathrm{M})$ at $80^{\circ} \mathrm{C}$, the $(Z)$-ester (14) was no longer detected. The product ratio, $28 \%(13)$ and $72 \%(15)$, turned out to be independent of the methanol concentration. This non-observation of (14) is attributable to the decreased methanol concentration, i.e., $k_{2}[\mathrm{MeOH}]^{3}$ (rate law for alcoholysis of ketens) ${ }^{6}$ becomes small compared with $k_{3}$. The independence of $(\mathbf{1 3}):(\mathbf{1 5})$ on the methanol concentration leaves no doubt that $k_{-1}^{\prime}<<k_{2}^{\prime}[\mathrm{MeOH}]^{3}$; hence the ring opening $(\mathbf{1 0}) \rightarrow(\mathbf{1 2})$ is irreversible under these reaction conditions.

\section{TABLE}

$(Z):(E)$-Vinylketen from

$\begin{array}{lcc} & (\mathbf{1 0}) & (\mathbf{1 6}) \\ \text { Thermolysis }\left(80^{\circ} \mathrm{C}\right) & 30: 70 & 40: 60 \\ \text { Photolysis }\left(10^{\circ} \mathrm{C}\right) & 40: 60 & 42: 58\end{array}$

It has been reported that heating of 2,4-dichloro-3-phenylcyclobut-2-enone with methanol yields stereospecifically methyl (E)-2,4-dichloro-3-phenylbut-3-enoate while the photolysis is stereospecific in $(Z)$-ester formation ${ }^{8}$ although the assignments were not convincing. A similar selectivity may occur in the ring opening of $(\mathbf{1 0})$ or the ethyl derivative (16). The irradiation of $(\mathbf{1 0})$ in methanol with a medium pressure mercury lamp (Hanau $Q 81$, glass filter) for $15 \mathrm{~min}$ at $10^{\circ} \mathrm{C}(67 \%$ conversion) resulted in the formation of $10 \%(13), 30 \%(14)$ and $60 \%(15)$. In a control experiment with $10 \mathrm{~min}$ irradiation time, an interconversion of the $(Z)$ - and $(E)$-esters (14) and (15) could not be detected. Thus, the photolysis of $(\mathbf{1 0})$ produced the vinylketens (11) and (12) in a 40:60 ratio. The data in the Table show that neither the thermolysis nor the photolysis of (10) and (16) proceeds with a high degree of stereoselectivity.

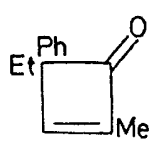

(16)

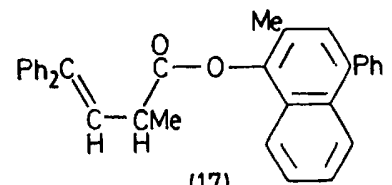

(17)
The vinylketen (6) cannot be intercepted with acetic acid or by $2+2$ cycloaddition with ethyl vinyl ether and the naphthol (9) was formed exclusively. The cyclobutenone (3) in triethylamine solution, however, is converted in 4 weeks at $25{ }^{\circ} \mathrm{C}$ into the naphthyl ester (17) in a $60 \%$ yield. Thus, the naphthoxide anion appears to be an efficient trapping agent for the vinylketen (6).

(Received, 20th October 1975; Com. 1185.)

1 E. F. Jenny and J. D. Roberts, J. Amer. Chem. Soc., 1956, 78, 2005; E. F. Silversmith, Y. Kitahara, M. C. Caserio, and J. D. Roberts, ibid., 1958, 80, 5840.

${ }^{2} \mathrm{H}$. Mayr and R. Huisgen, Angew Chem. Internat. Edn., 1975, 14, 499.

${ }^{3}$ H. Mayr, Angew. Chem. Internat. Edn., 1975, 14, 500.

${ }^{4}$ For statistical analysis of substituent effects in the n.m.r. spectra of 3-alkoxycyclobutanones see H. Mayr and R. Huisgen, Tetrahedron Letters, $1975,1349$.

5 Review: S. Hünig and H. Hoch, Fortschr. Chem. Forsch., 1970, 14, 235.

${ }^{6}$ P. J. Lillford and D. P. N. Satchell, J. Chem. Soc. $(B), 1968,889$.

${ }^{7}$ C. Pascual, J. Meier, and W. Simon, Helv. Chim. Acta, 1966, 49, 164.

${ }^{8} \mathrm{~J}$. E. Baldwin and M. C. McDaniel, J. Amer. Chem. Soc., 1968, 90, 6118. 